\title{
Letter \\ How much sleep apnea is too much? Authors' response
}

\author{
David M Rapoport ${ }^{1}$, Fumiharu Togo ${ }^{2}$ and Benjamin H Natelson ${ }^{3}$
}

\begin{abstract}
1'Department of Medicine, Division of Pulmonary and Critical Care Medicine, NYU School of Medicine, New York, NY 10016, USA
${ }^{2}$ Department of Work Stress Control, Japan National Institute of Occupational Safety and Health, Kawasaki, 214-8585, Japan

${ }^{3}$ Pain and Fatigue Study Center, Department of Pain Medicine and Palliative Care, Beth Israel Medical Center, New York, NY 10003, USA
\end{abstract}

Corresponding author: Benjamin H. Natelson, bnatelson@bethisraelny.org

Published: 15 July 2009

Arthritis Research \& Therapy 2009, 11:410 (doi:10.1186/ar2691)

This article is online at http://arthritis-research.com/content/11/4/410

(c) 2009 BioMed Central Ltd

See related research by Togo et al., http://arthritis-research.com/content/10/3/R56, and related letter by Martinez et al., http://arthritis-research.com/content/11/4/410

Our study [1] had two main purposes: first, to examine the occurrence of sleep-disordered breathing (SDB) in patients with chronic fatigue syndrome (CFS), and then to exclude patients with this confound in order to evaluate the structure of sleep in a group of patients with "pure" CFS to look for relations with the symptoms of fatigue and sleepiness. In order to do this, we had to define an upper limit for the occurrence of respiratory events that might explain the daytime symptoms. Martinez and Cassol are correct that the currently accepted cutpoint for abnormality proposed by the American Academy of Sleep Medicine (AASM) and Centers for Medicare \& Medicaid Services (CMS) in the US is an apnea-hypopnea index $(\mathrm{AHI}) \geq 5$ per hour but less than 15 per hour - but only if symptoms such as EDS are present. However, the $\mathrm{AHI}$ is an insensitive measure of the severity of mild respiratory events (such as respiratory event-related arousals (RERAs)) as it requires either a 3 or $4 \%$ desaturation for the definition of hypopnea and this is frequently not present in respiratory events that cause arousal and may contribute to sleep disruption (RERA). For this reason, our cutpoint for SDB is based on a respiratory disturbance index (RDI) of 18 events per hour and not the $A H I$. The RDI includes RERAs along with the AHI, although we used a slightly different definition of hypopneas beginning with any event showing "flow limitation" rather than the current amplitude based entry criterion. The rationale for this is from an earlier paper by our group [2] that shows that the sensitivity and specificity for explaining EDS in a group composed of symptomatic but mild SDB and noncomplaining controls were greater using the RDI criterion than the AHI criterion. Since that paper, it has become customary to use a cut-off of 15 events per hour for RDI in many applications. It is important to note that for RDI, using 5 events per hour will include essentially $80 \%$ of the normal population. Furthermore, as pointed out in our current paper [1], although we chose 18 events per hour as a cut-off to exclude three subjects (one with CFS and two normal subjects) when defining the final group for our sleep analysis who might have SDB, in fact the highest RDls we saw in the non-SDB group were 10.4, 10.8, 10.1, and 9.5 events per hour; we feel these numbers are within a range seen in many normal subjects without EDS [2]. Thus, our cut-off of 18 is not effectively different from the more recently accepted number of 15 events per hour. And after excluding those subjects with high RDI values, there was no significant difference in the $\mathrm{AHI}$ of patients with only CFS, those with CFS and fibromyalgia and healthy controls $(1.7 \pm 1.6$, $2.8 \pm 2.6$, and $2.2 \pm 1.7$, respectively).

In fact, the above logic is not fundamentally different from the choice made by Martinez and Cassol in their own work [3-6], where they define SDB as present in fibromyalgia when the $\mathrm{AHI}<5$ and when more than 10 RERAs per hour occurred $(5+10$ events $=15$ per hour, similar to our cut-off of 18 per hour).

\section{Competing interests}

DMR has received grant funding from Fisher Paykell Healthcare, Ventus Medical, Restore Medical, Advanced Brain Monitoring, SleepEx. DMR holds multiple patents to treatment of SDB with nasal CPAP - licensed to Fisher Paykel Healthcare and Covidian and receives royalties from these. FT and BHN have no competing interests to declare.

\section{References}

1. Togo F, Natelson BH, Cherniack NS, FitzGibbons J, Garcon C Rapoport DM: Sleep structure and sleepiness in chronic fatigue syndrome with or without coexisting fibromyalgia. Arthritis Res Ther 2008, 10:R56.

$\mathrm{AHI}=$ apnea-hypopnea index; $\mathrm{CFS}=$ chronic fatigue syndrome; $\mathrm{RDI}=$ respiratory distress index; RERA = respiratory event-related arousal; SDB = sleep-disordered breathing. 
2. Ayappa I, Norman RG, Krieger AC, Rosen A, O'malley RL, Rapoport DM: Non-invasive detection of respiratory effortrelated arousals (RERAs) by a nasal cannula/pressure transducer system. Sleep 2000, 23:763-771.

3. Germanowicz D, Lumertz MS, Martinez D, Margarites AF: Sleep disordered breathing concomitant with fibromyalgia syndrome. J Bras Pneumol 2006, 32:333-338.

4. Martinez-Lavin M: Biology and therapy of fibromyalgia: Stress, the stress response system, and fibromyalgia. Arthritis Res Ther 2007, 9:216.

5. Félix FHC, Fontenele JB: Is fibromyalgia a cardiovascular disease? A comment on Martinez-Lavin's review 'Stress, the stress response system, and fibromyalgia'. Arthritis Res Ther 2007, 9:404.

6. Martinez D, Cassol CM: Fibromyalgia and sleep-disordered breathing: the missing link. Arthritis Res Ther 2008, 10:408. 